\title{
23 Emotionsregulation im Kontext von Stereotype Threat: Die Reduzierung der Effekte negativer Stereotype bei ethnischen Minderheiten
}

\author{
Sarah E. Martiny, Thomas Götz und Melanie Keller
}

Viele der in Deutschland lebenden Menschen mit Migrationshintergrund haben Schwierigkeiten mit einer sogenannten „erfolgreichen Integration“ in die deutsche Gesellschaft. Dies zeigt sich nach Meinung zahlreicher Experten vor allem bei ihren Leistungen im deutschen Bildungssystem und auf dem deutschen Arbeitsmarkt. Das Institut für Sozialforschung und Gesellschaftspolitik stellte in seinem Bericht „Integration in Deutschland - Erster Integrationsindikatorenbericht" im Jahr 2009 fest, dass im Schuljahr 2006/2007 16\% der Schüler(innen) mit Migrationshintergrund die Schule ohne Abschluss verließen, während dies nur bei 6,5\% der deutschen Schüler(innen) ohne Migrationshintergrund der Fall war. Ein ähnliches Bild zeigt sich bei den Arbeitslosenzahlen: während 2007 10,1\% der Deutschen ohne Migrationshintergrund arbeitslos gewesen sind, waren dies doppelt so viele Menschen mit Migrationshintergrund (20,3\%). Standardisierte Tests, welche die Leistungen von Schüler(innen) mit und ohne Migrationshintergrund vergleichen, kommen zu ähnlichen Ergebnissen: So deckten die Ergebnisse von PISA 2003 und 2006 soziale Disparitäten auf und zeigten, dass Deutschland eines derjenigen Länder ist, in dem eine der größten Diskrepanzen zwischen den Leistungen von Schüler(innen) mit und ohne Migrationshintergrund zu finden ist (Prenzel u. a. 2004, 2007). Die Ergebnisse von PISA 2009 zeigten zwar gerade im Hinblick auf die Lesekompetenz, dass eine signifikante Leistungssteigerung für Jugendliche mit Migrationshintergrund seit PISA 2000 stattgefunden hat, doch gleichzeitig sind weiterhin große Disparitäten verbunden mit Migrationsgeschichte beobachtbar.

Diese alarmierenden Ergebnisse haben die öffentliche Debatte über die Gründe der niedrigen Leistungen von Menschen mit Migrationshintergrund in Deutschland wieder entfacht. Weit verbreitete Annahmen gehen davon aus, dass die geringen Leistungen der Menschen mit Migrationshintergrund auf zwei wesentliche, miteinander in Zusammenhang stehende Ursachen zurückzuführen sind: mangelnde Kenntnisse der deutschen Sprache und niedriger sozio-ökonomischer Status der Familien. Die Ergebnisse von PISA 2003 zeigten allerdings, dass unter Berücksichtigung dieser beiden Faktoren (statistische Kontrolle) der Unterschied zwischen den Leistungen der Schüler(innen) mit und ohne Migrationshintergrund zwar abnimmt, aber weiterhin bedeutsam bleibt (d. h. 
er bleibt statistisch signifikant). Dies bedeutet, dass weitere Faktoren für die niedrigen Leistungen von Schüler(innen) mit Migrationshintergrund verantwortlich sein müssen.

Mitte der neunziger Jahre des vergangenen Jahrhunderts konnten Claude Steele und Joshua Aronson (1995) zeigen, dass die niedrigen Leistungen bestimmter sozialer Gruppen durch den Einfluss leistungsbezogener negativer Stereotype verursacht werden können. So zeigten die beiden Autoren zum Beispiel, dass Afro-Amerikaner(innen), die ihre Ethnizität vor der Bearbeitung eines verbalen Leistungstest angeben mussten, schlechter abschnitten, als Afro-Amerikaner(innen), die ihre Ethnizität nicht vor der Testbearbeitung angeben mussten. Die Autoren folgerten daraus, dass die Angabe der Ethnizität das negative Stereotyp („Afro-Amerikaner(innen) sind weniger intelligent als weiße Amerikaner(innen)" ), das über Afro-Amerikaner(innen) und verbale Intelligenz besteht, aktiviert hatte (Steele \& Aronson, 1995, Studie 4). Dieses als Stereotype Threat bezeichnete Phänomen bedeutet also, dass das bloße Aktivieren negativer Stereotype dazu führen kann, dass die betroffenen Gruppenmitglieder einen signifikanten Leistungsabfall zeigen. Innerhalb der vergangenen zwei Jahrzehnte konnten Stereotype-ThreatEffekte in mehr als 200 Studien und in einer Vielzahl von Bereichen (bzw. Domänen) und Gruppen gezeigt werden: unter anderem bei Afro-Amerikaner(inne)n und verbaler Intelligenz (z. B. Blascovich, Spencer, Quinn \& Steele, 20o1; Steele \& Aronson, 1995), Frauen und Mathematik (z. B. Keller, 2002; Keller \& Dauenheimer, 2003; Schmader \& Johns, 2003), Schüler(innen) mit niedrigem sozio-ökonomischen Status und Leistungen (z. B. Croizet \& Claire, 1998; Spencer \& Castano, 2007) und bei älteren Menschen und Gedächtnis (z. B. Levy, 1996; Rahhal, Hasher \& Colcombe, 20o1; für einen Überblick siehe Martiny \& Götz, 2011).

Es liegt somit nahe anzunehmen, dass die Aktivierung negativer, sich auf die Leistungsfähigkeit ethnischer Minderheiten beziehende Stereotype, bei diesen zu leistungseinschränkenden Effekten führt. Da negative Emotionen im Kontext von Stereotype Threat eine bedeutsame Rolle spielen (z. B. Versagensängste, Hoffnungslosigkeit, Ärger) sollen im folgenden Kapitel Ansätze zur Emotionsregulation in die Forschung zu Stereotype Threat integriert werden. Dazu wird zunächst ein Überblick über die bestehende Forschung zu Stereotype Threat gegeben, bei dem immer wieder Bezug auf die besondere Situation ethnischer Minderheiten genommen wird. Dann werden verschiedene Arten der Emotionsregulation dargestellt, die von Menschen mit Migrationshintergrund in Stereotype-Threat-Situationen eingesetzt werden können (selbstverständlich auch von Menschen ohne Migrationshintergrund). 


\section{Empirische Befunde zur Wirkung der Aktivierung negativer Stereotype}

\section{Effekte auf Leistung}

Es liegt eine Vielzahl an Studien vor, in welchen die Wirkung der Aktivierung negativer Stereotype im Labor untersucht wurde und deren Ergebnisse konsistent einen Leistungsabfall der vom Stereotyp betroffenen Gruppenmitglieder zeigen (z. B. Croizet \& Claire, 1998; Keller, 2002; Keller \& Dauenheimer, 2003; Schmader \& Johns, 2003; Steele \& Aronson, 1995). In diesen Studien wurden verschiedene Formen der Aktivierung negativer Stereotype gewählt. So wurde zum Beispiel in der ersten Veröffentlichung zu Stereotype Threat von Steele und Aronson (1995) der Fokus auf die Konsequenzen der Aktivierung negativer Stereotype über ethnische Minderheiten gelegt. In der Arbeit wurde die Leistung von Afro-Amerikaner(inne)n und weißen Amerikaner(inne)n in Bezug auf ihre verbalen Fähigkeiten untersucht. Dabei wurden zwei verschiedene Arten der Aktivierung des negativen Stereotyps verwendet. In den ersten drei Studien manipulierten die Autoren die Diagnostizität des Tests (also ob der Test Intelligenz misst). In der vierten Studie aktivierten die Autoren das negative Stereotyp subtiler und realitätsnäher, indem die Teilnehmenden in der Experimentalbedingung lediglich ihre Ethnizität vor der Aufgabenbearbeitung angeben mussten. Diese Form der Aktivierung eines negativen Stereotyps reichte aus, um zu einer Beeinträchtigung der Leistung der AfroAmerikaner(innen) in der Experimentalbedingung zu führen. Die Effektivität dieser und zahlreicher anderer Stereotyp-Manipulation wurde inzwischen in zahlreichen Studien repliziert (Ambady, Shih, Kim, \& Pittinsky, 2001; McGlone \& Aronson, 2006; Shih, Pittinsky, \& Ambady, 1999; Shih, Pittinsky, \& Trahan, 2006; Yopyk \& Prentice, 2005).

In neueren Arbeiten ist vermehrt versucht worden, die im Labor gefunden Effekte im Feld zu replizieren. So liegen beispielsweise inzwischen mehrere Studien vor, die Stereotype Threat in Schulen demonstrieren. Keller und Kollegen (Keller \& Dauenheimer, 2003; Keller, 2007) zeigten leistungseinschränkende Effekte in Folge der Aktivierung negativer Stereotype im Klassenzimmer bei Mädchen in Mathematik. Huguet und Régner (2007) demonstrierten in zwei weiteren Studien die leistungseinschränkenden Effekte der Aktivierung negativer Stereotype bei Mädchen im Klassenzimmer, wenn sie glaubten, dass ein Test mathematische Fähigkeiten erfasst. Dies bedeutet, dass die negativen Konsequenzen der Aktivierung von Stereotypen sowohl im Labor als auch im Feld zu beobachten sind.

Bislang liegen unseres Wissens keine Studien vor, welche die direkten Konsequenzen des Erlebens von Stereotype Threat auf Leistungen bei ethnischen Minderheiten in Deutschland untersucht haben. Aufgrund der konsistenten Befunde aus den USA zu den Wirkungsweisen negativer Stereotype und der Tatsache, dass in Deutschland negative Stereotype über bestimmte Migrantengruppen weit verbreitetet $\operatorname{sind}(\mathrm{z}$. B. über türkisch-stämmige Migranten, siehe dazu Kahraman \& Knoblich, 20oo) erscheint es sehr wahrscheinlich, dass auch in Deutschland lebende ethnische Minderheiten in ihren 
Leistungen durch die Aktivierung negativer, leistungsbezogener Stereotype behindert werden können. So könnte es zum Beispiel in der Schule der Fall sein, dass alleine durch das Wissen, dass der nachfolgende Test diagnostisch für akademische Fähigkeiten ist, negativen Stereotype, die über türkisch-stämmige Menschen verbreitet sind, aktiviert werden. Diese Stereotypaktivierung könnte dann im Folgenden zu einem Leistungsabfall der betroffenen Schüler(innen) führen.

\section{Effekte auf Motivation}

Es liegen bisher nur wenige Stereotype-Threat-Studien vor, in denen die Konsequenzen für motivationale Variablen untersucht wurden. Hierbei ist es wichtig zu erwähnen, dass Motivation sowohl eine Konsequenz als auch einen Mediator im Kontext von Stereotype Threat darstellt (siehe Abb. 13). Im folgenden Abschnitt werden motivationale Konsequenzen der Stereotypaktivierung dargestellt.

Abbildung 13 Modell zur Wirkung von Moderatoren und Mediatoren des Effekts von Stereotype Threat auf Leistung und Motivation

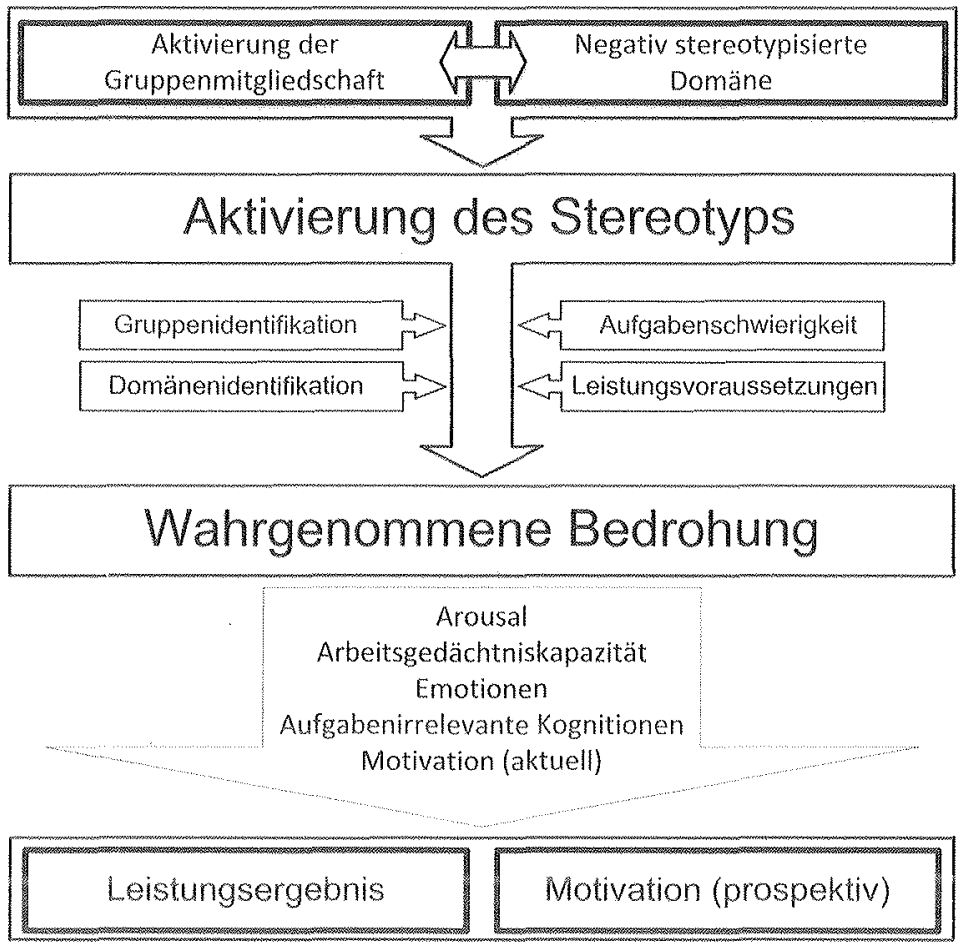


Eine Studie von Koch, Müller und Sieverding (2008) konnte zeigen, dass die Aktivierung negativer Stereotype die Wahrscheinlichkeit internaler Attribution von Misserfolg erhöht (z. B. „Ich bin einfach nicht gut genug, um diese Aufgabe erfolgreich zu lösen“). Darüber hinaus gibt es Studien, welche die Effekte der Aktivierung negativer Stereotype auf SelfHandicapping-Strategien untersuchen. Self-Handicapping-Strategien sind definiert als jene Handlungen oder Wahlen von Leistungssettings, welche dazu führen, dass selbstbezogene Fähigkeitsattributionen vermieden werden können (Berglas \& Jones, 1978). Dies bietet zum Beispiel die Möglichkeit, Misserfolg external zu attribuieren („Es lag nicht an meinen Fähigkeiten, dass ich die Aufgaben nicht lösen konnte, sondern daran, dass die Aufgaben unfair waren"). Es handelt sich also um defensive Strategien, die dazu führen, dass in der Regel ungünstige Lern- und Leistungsbedingungen geschaffen werden, die dann als Ursachenzuschreibungen für die niedrigen Leistungen dienen können (z. B. Aufgaben werden weniger geübt als notwendig). Somit geht Self-Handicapping häufig mit niedrigen Leistungen einher. Wenn es dann unter diesen ungünstigen Bedingungen zu einem Misserfolg kommt, kann der Misserfolg auf die Umstände, d. h. external, attributiert werden (im zuvor genannten Beispiel: die mangelnde Übung); wenn es jedoch zum Erfolg kommt, kann dieser umso mehr auf die eigenen Fähigkeiten, d.h. internal, attribuiert werden („Ich bin so begabt, dass ich es geschafft habe, obwohl ich nicht geübt habe"). Es liegen Studien vor, die zeigen, dass die Aktivierung negativer Stereotype Self-Handicapping erhöht. Stone (2002) untersuchte zum Beispiel die Leistungen von weißen männlichen Amerikanern beim Golfspielen, wobei das Stereotyp aktiviert wurde, dass Afro-Amerikaner über mehr natürliche athletische Fähigkeiten verfügen als weiße Amerikaner (Experimentalgruppe). Es zeigte sich, dass Weiße in der Experimentalgruppe, die Golfspielen wertschätzten, weniger übten als Studienteilnehmende in der Kontrollgruppe und weniger als Studienteilnehmende in der Experimentalgruppe, die Golfspielen nicht wertschätzten. Diese Ergebnisse werden von dem Autor als Hinweis interpretiert, dass das Aktivieren negativer Stereotype Self-Handicapping erhöht.

Eine weitere motivationale Konsequenz der Aktivierung negativer Stereotype ist Disengagement, d. h. das Sich-Lossagen bzw. das Sich-Distanzieren von einer bestimmten Domäne. Sind Individuen wiederholt negativen Stereotypen ausgesetzt, so steigt das Risiko, dass sie sich von der Domäne, in der sie mit negativen Stereotypen konfrontiert werden, (temporär) distanzieren. Je besser es ihnen gelingt, sich von der Domäne $\mathrm{zu}$ distanzieren, desto weniger ist ihr Selbstkonzept dann noch von den Leistungen in der jeweiligen Domäne abhängig. Je weniger das Selbstkonzept einer Person von ihrer Leistung in der Domäne abhängig ist, desto weniger ist die Person motiviert und engagiert, gute Leistungen in der jeweiligen Domäne zu erbringen. So zeigten zum Beispiel von Hippel et al. (2005), dass weiße Amerikaner(innen) in den Fällen, in denen sie davon ausgingen, dass ihre Ergebnisse in dem nachfolgenden IQ-Test mit denen von Asiat(inn)en verglichen werden würden (aktiviertes Stereotyp: Asiat(inn)en sind intelligenter als Weiße), sich eher von der Domäne (hier Intelligenz) distanzierten. Eingeschränktes und zeitlich begrenztes Disengagement kann neben den negativen Effek- 
ten, wie reduzierte Motivation, sich in dem jeweiligen Bereich anzustrengen, aber auch positive Effekte haben (Nussbaum \& Steele, 2007). Dies bedeutet, dass es sich bei Disengagement um eine sinnvolle kurzfristige Strategie handeln kann, die dazu beiträgt, dass Menschen, die mit negativen Stereotypen konfrontiert sind, keine dauerhaften Schäden an ihren Selbstkonzepten und ihrer Motivation erleiden.

Neben dem kurzfristigen Disengagement können sich Menschen aber auch langfristig von einer Domäne distanzieren - dies wird als Disidentifkation bezeichnet. Davies, Spencer, Quinn, \& Gerhardstein (2002) fanden in einer ersten Studie, dass das Betrachten geschlechtsstereotyper Werbung die Leistungen der teilnehmenden Frauen in einem Mathematiktest reduzierte. In einer weiteren Studie fanden die Autor(inn)en Belege dafür, dass Frauen im Anschluss an das Betrachten geschlechterstereotyper Werbung weniger Interesse an naturwissenschaftlichen als an geisteswissenschaftlichen Berufsfeldern und Studienfächern äußerten, als Frauen, die geschlechtsneutrale Werbung sahen.

Die beschriebenen Befunde, die darauf hinweisen, dass das Aktivieren negativer Stereotype auch Konsequenzen für langfristige leistungsmotivationale Variablen haben kann, sind im Zusammenhang mit den bekannten Schwierigkeiten bestimmter ethnischer Minderheiten im deutschen Bildungssystem von zentraler Bedeutung. Die Tatsache, dass das wiederholte Erleben von Stereotype Threat (kumulative Effekte) neben direkten Effekten auf Leistungen auch zu einer reduzierten Motivation, sich in bestimmten (akademischen) Bereichen anzustrengen, führen kann, kann eventuell als eine weitere, indirekte Erklärung für die schlechten schulischen Leistungen bestimmter ethnischer Gruppen herangezogen werden.

\section{Mediatoren und Moderatoren im Kontext von Stereotype Threat}

Eine zentrale Frage, die in den vergangenen Jahren viel Forschung nach sich gezogen hat, beschäftigt sich mit den Prozessen, welche zwischen der Aktivierung eines negativen Stereotyps und den daraus resultierenden Konsequenzen liegen, also welches die Mediatoren des Zusammenhangs sind. Trotz der vielen Forschungsarbeiten und der Tatsache, dass es sich bei Stereotype Threat um ein in vielen Bereichen bzw. Domänen auftretendes Phänomen handelt, konnte die Forschung die zugrunde liegenden Prozesse bisher noch nicht eindeutig klären. Darüber hinaus wird im Folgenden dargestellt, welche Faktoren die negativen Wirkungen der Aktivierung negativer Stereotype erhöhen und welche sie reduzieren - es wird somit auf die Moderatoren des Zusammenhangs der Aktivierung von Stereotypen und entsprechender Konsequenzen eingegangen. 


\section{Mediatoren}

Basierend auf der Arbeit von Schmader, Johns \& Forbes (2008) wird davon ausgegangen, dass Stereotype Threat primär auf einer Reduktion der Kapazitäten des Arbeitsgedächtnisses basiert. So untersuchten beispielsweise Schmader und Johns (2003), ob das Arbeitsgedächtnis von der Aktivierung negativer Stereotype beeinflusst wird. Es zeigte sich, dass die Unterschiede in der Arbeitsgedächtniskapazität den Zusammenhang zwischen Stereotypaktivierung und Leistung mediierten. Aus diesem Grund wird im Folgenden im Detail auf spezifische und empirisch belegte Mediatoren eingegangen, die mit reduzierten Kapazitäten des Arbeitsgedächtnisses einhergehend zu den beobachteten Leistungseinbußen in Folge der Aktivierung negativer Stereotype beitragen (siehe Abbildung 13).

Physiologische Erregung (Arousal). Croizet et al. (2004) zeigten, dass die Aktivierung negativer Stereotype zu einer erhöhten Herzratenvariabilität führt, welche einen physiologischen Indikator für mentale Belastung darstellt. In weiteren Studien wurde demonstriert, dass Menschen nach der Aktivierung negativer Stereotype eine erhöhte Hautleitfähigkeit und einen erhöhten Blutdruck haben (z. B. Osborne, 2007). Blascovich et al. (2001) zeigten weiterhin, dass der Blutdruck bei der Aktivierung negativer Stereotype schneller anstieg und im Vergleich zu Personen der Kontrollgruppe auf einem höheren Niveau blieb. Beilock und Kollegen (Beilock, Rydell \& McConnell, 2007; Rydell, McConell, \& Beilock, 2009) fanden Belege, dass die Aktivierung negativer Stereotype phonologische Komponenten des Arbeitsgedächtnisses beeinträchtigt - diese unterstützen das Denken bei komplexen kognitiven Aktivitäten (Carlson, 1997). Schließlich konnten Martiny, Roth und Deaux (2011) Arousal als Mediator des Zusammenhangs zwischen der Aktivierung eines negativen Stereotyps und reduzierten Leistungen sogar anhand eines Selbsteinschätzungsmaßes nachweisen.

Aufgabenirrelevante Kognitionen. In Zusammenhang mit der Annahme, dass das Aktivieren negativer Stereotype zu erhöhtem physiologischen Arousal führt, steht die Annahme, dass die aktivierten Stereotype auch aufgabenirrelevante Gedanken erzeugen. So zeigten bereits Steele und Aronson (1995), dass das Aktivieren eines negativen Stereotyps zu einer erhöhten Zugänglichkeit von stereotypen Kognitionen führt. Weiterhin konnte in einer Studie von Cadinu, Maass, Rosabianca und Kiesner (2005) gezeigt werden, dass das Aktivieren eines negativen Stereotyps vor der Bearbeitung mathematischer Probleme bei Frauen zu geringeren Leistungen führte. Diese Leistungseinbußen wurden durch die Anzahl domänenspezifischer negativer Gedanken (z. B. „Ich bin einfach nicht gut in Mathe") mediiert. In einer psycho-physiologischen Studie wurde der Zusammenhang zwischen aufgabenirrelevanten Kognitionen, Emotionen und Leistung genauer untersucht. Die Autor(inn)en (Krendl, Richeson, Kelley \& Heatherton, 2008) demonstrierten, dass Frauen, die mathematische Probleme bei gleichzeitiger Aktivierung negativer Stereotype lösen mussten, eine erhöhte Aktivität in den Gehirnarealen aufwiesen, die im Zusammenhang mit der Verarbeitung negativer Informationen stehen 
(ventral anterior cingulate cortex; $v A C C$ ). Frauen in der Kontrollbedingung hingegen zeigten eine erhöhte Aktivität in den Arealen, die mit Mathematiklernen und Problemlösen in Verbindung stehen.

Motivation. In Bezug auf Leistungserwartungen als eine zentrale motivationale Variable liegen empirische Belege vor, die darauf hinweisen, dass diese durch die Aktivierung negativer Stereotype negativ beeinflusst werden können. So konnte gezeigt werden, dass das Aktivieren von Geschlechterstereotypen dazu führt, dass Frauen ihre Leistung in einer nachfolgenden Aufgabe unterschätzen. Dieser Effekt konnte bei Frauen für räumliche Wahrnehmung (Stangor, Carr, \& Kiang, 1998) und für Verhandlungsaufgaben gezeigt werden (Kray, Thompson \& Galinski, 2001) werden. Darüber hinaus zeigten Cadinu und Kollegen (Cadinu, Maass, Frigerio, Impagliazzo, \& Latinotti, 2003, Studie 1), dass Frauen, die Mathematik sehr wertschätzen, nach der Aktivierung negativer Stereotype reduzierte Leistungserwartungen zeigten, die sich dann auch auf die nachfolgenden Leistungen auswirkten. In einer zweiten Studie fanden die Autor(inn)en sogar, dass Leistungserwartungen den Zusammenhang von Stereotypaktivierung und Leistung vollständig mediierten.

Emotionen. Schon früh wurde vermutet, dass negative Emotionen eine zentrale Rolle bei den durch die Aktivierung negativer Stereotype hervorgerufenen Leistungseinschränkungen spielen (Steele, 1997). Seitdem haben zahlreiche Studien die Rolle von (ïberwiegend negativen) Emotionen während und nach der Aufgabenbearbeitung im Anschluss an die Aktivierung negativer Stereotype untersucht. In einigen Studien konnte gezeigt werden, dass die Aktivierung negativer Stereotype in der Tat negative Emotionen auslöst (z. B. Bosson, Haymovitz, \& Pinel, 2004; Keller \& Dauenheimer, 2003; Spencer, Steele, \& Quinn, 1999). So verglichen zum Beispiel Bosson et al. (2004) homosexuelle und heterosexuelle Männer, die mit Vorschulkindern interagierten. Sobald bei den Homosexuellen ein negatives Stereotyp bezüglich ihres Umgangs mit Kindern aktiviert wurde ( $z$. B. homosexuelle Männer können schlecht mit Kindern umgehen), zeigten sie mehr nonverbale Ängstlichkeit als Homosexuelle in der Kontrollgruppe. Interessanterweise unterschieden sich die beiden Gruppen jedoch nicht in ihrer selbstberichteten Ängstlichkeit. Darüber hinaus fand eine Studie von Keller und Dauenheimer (2003), dass negative Emotionen den Zusammenhang von Stereotypaktivierung und Leistung mediierten. Allerdings gelang es nicht immer, Zusammenhänge zwischen negativen Emotionen und reduzierten Leistungen im Kontext von Stereotype Threat nachzuweisen (z. B. Beilock et al., 2007; Cadinu et al., 2005; Schmader \& Johns, 2003; Steele \& Aronson, 1995; Stone, Lynch, Sjomeling \& Darley, 1999). Die teilweise inkonsistenten empirischen Ergebnisse in Bezug auf die Rolle der Emotionen beim Erleben von Stereotype Threat lassen sich vermutlich auf mehrere Aspekte zurückführen. So wurden in den meisten Studien nur wenige Emotionen (meist Angst und gelegentlich Frustration) erhoben und diese auch nur zu einer bestimmten Zeit (entweder direkt vor oder direkt nach der Aufgabenbearbeitung). Es liegt nahe zu argumentieren, dass das Messen mehrerer Emotionen zu mehreren Zeitpunkten sinnvoll wäre. 


\section{Moderatoren}

Im Folgenden werden vier zentrale Moderatoren vorgestellt, die aus theoretischer Perspektive überzeugend sind und zu welchen kumulative empirische Evidenz vorhanden ist (siehe Abbildung 13).

Aufgabenschwierigkeit. Als einer der zentralen Moderatoren des Zusammenhangs zwischen der Aktivierung negativer Stereotype und den daraus resultierenden negativen Konsequenzen hat sich die Aufgabenschwierigkeit erwiesen. Die negativen Effekte von Stereotypaktivierung zeigen sich nur bei schwierigen Aufgaben. So ließ Keller (2007) zum Beispiel Frauen und Männer eine Reihe von Mathematikaufgaben unterschiedlichen Schwierigkeitsgrades bearbeiten. In der Bedingung, in welcher ein negatives Stereotyp über Frauen und Mathematik aktiviert wurde, zeigten die Studienteilnehmerinnen reduzierte Leistungen - allerdings nur bei schwierigen Mathematikaufgaben. Reduzierte Leistungen aufgrund erhöhter Aufgabenschwierigkeit lassen sich mit Hilfe der weiter oben angeführten Mediationsprozesse im Kontext von Stereotype Threat erklären. Es kann davon ausgegangen werden, dass im Falle der Aktivierung eines negativen Stereotyps kognitive Ressourcen beansprucht werden, um in irgendeiner Form das negative Stereotyp zu bewältigen. Diese kognitiven Ressourcen stehen dann nicht mehr für die Aufgabenbearbeitung zur Verfügung. Vor allem bei schwierigen Aufgaben, die alle zur Verfügung stehenden kognitiven Ressourcen benötigen, sollte dies leistungsschädlich wirken. Auch das emotionale Erleben und die Motivation (z. B. Leistungserwartungen; siehe oben) können von der Schwierigkeit der Aufgabe beeinflusst werden: Je schwieriger eine Aufgabe ist, desto wahrscheinlicher werden beispielsweise spezifische negative Emotionen (z. B. Angst, Scham, Hoffnungslosigkeit) aktiviert und desto wahrscheinlicher entstehen negative Leistungserwartungen. Auch in Bezug auf die Gruppe der Menschen mit Migrationshintergrund bedeutet dies, dass die Wirkung der Aktivierung negativer Stereotype vermutlich vor allem bei subjektiv als schwierig wahrgenommenen Aufgaben auftritt, denn in diesem Fall sollte die Leistung besonders stark durch die eingeschränkten Kapazitäten des Arbeitsgedächtnisses beeinflusst werden.

Identifikation mit der Gruppe. Ein weiterer moderierender Faktor ist die Identifikation mit der Gruppe. Je stärker sich ein Gruppenmitglied mit der jeweils salienten Gruppe identifiziert, desto stärkeren Einfluss hat die Evaluation der Gruppe auf das Selbstkonzept des Gruppenmitglieds. Dies erklärt, warum die negative Wirkung des Aktivierens eines negativen Stereotyps umso stärker ist, je mehr sich die Gruppenmitglieder mit ihrer Eigengruppe identifizieren. Nur wenn sich ein Gruppenmitglied mit der vom Stereotyp betroffenen Gruppe identifiziert, beeinflusst der Wert der Gruppe auch das Selbstkonzept des Gruppenmitglieds (Tajfel \& Turner, 1979). Und nur wenn der Wert der Gruppe das Selbstkonzept des Individuums beeinflusst, stellt eine Bedrohung der Gruppe durch die Aktivierung negativer Stereotype auch eine Bedrohung für das Selbstkonzept des individuellen Gruppenmitglieds dar. Mit der Gruppe hoch identifizierte Gruppenmitglieder werden also versuchen, ihr Selbstkonzept vor den nega- 
tiven, u. a. selbstwertschädlichen Effekten der negativen Stereotype zu schützen. Die erhöhte Vulnerabilität hoch identifizierter Gruppenmitglieder gegenüber Stereotype Threat konnte mittlerweile in einer Reihe von Studien nachgewiesen werden ( $z$. B. Davis, Aronson, \& Salinas, 2006; Martiny, Roth, Jelenec, Steffens, \& Croizet, 2012; Schmader, 2002; Wout, Danso, Jackson, \& Spencer, 2008).

Auch dieser Moderator hat spezifische Implikationen für Menschen mit Migrationshintergrund. Wie die Arbeiten zum Rejection-Identification Modell (z. B. Branscombe, Ellemers, Spears, \& Doosje, 1999; Branscombe, Schmitt, \& Harvey, 1999; Jetten, Branscombe, Schmitt, \& Spears, 2001) u. a. demonstrierten, kann die Wahrnehmung von Diskriminierung dazu führen, dass die Identifikation mit der eigenen Gruppe erhöht wird. Dies bedeutet, dass es bei ethnischen Minderheiten in Folge wahrgenommener Diskriminierung zu einer starken Identifikation mit der Eigengruppe kommen kann, was dann bei der Aktivierung negativer Stereotype in Leistungssituationen zu besonders leistungseinschränkenden Effekten führen könnte.

Identifikation mit der Domäne. Das Ausmaß, zu welchem eine Person einer bestimmten Domäne Wert beimisst (d.h. Domänenidentifikation), hat auch Einfluss auf das Erleben von Stereotype Threat. Je wichtiger einer Person eine Domäne ist, desto stärker wirkt sich die Rückmeldung in dieser Domäne auf ihr Selbstkonzept aus (Aronson, 2002; Aronson et al., 1999; Cadinu et al., 2003; Keller, 2007, Levy, 1996; Leyens, Désert, Croizet, \& Darcis, 20oo; Spencer et al., 1999; Stone et al., 1999). Martiny et al. (2011) ist es in einem vollständig kontrollierten experimentellen Setting gelungen, die kausale Wirkung der Identifikation mit der Domäne im Kontext von Stereotype Threat empirisch zu belegen. Die Autorinnen zeigten, dass die Manipulation der Relevanz einer unbekannten Domäne dazu führt, dass nur diejenigen Personen einen signifikanten Leistungsabfall zeigten, welche die untersuchte Domäne als für sie wichtig einschätzten. Ähnlich wie bei der Identifikation mit der Gruppe kann davon ausgegangen werden, dass die Identifikation mit der Domäne auf die mediierenden Prozesse wirkt und dadurch Einfluss auf Leistungsergebnisse hat. Je wichtiger die Domäne, desto wahrscheinlicher ist es, dass durch die Aktivierung negativer Stereotype negative Emotionen ausgelöst werden.

Leistungsvoraussetzungen. Zu Individuen mit besonders günstigen Leistungsvoraussetzungen lassen sich auf der Basis des bisher Dargestellten eine Reihe theoretischer Annahmen formulieren. Allerdings gibt es hierzu im Kontext von Stereotype Threat noch relativ wenig Forschung. Häufig zeigt sich, dass eine starke Wertschätzung der Domäne zu erhöhter Anstrengung und dadurch zu guten Leistungen führt (Osborne, 1997; Osborne \& Walker, 2006; Steele, 1992). Wie bereits ausgeführt, konnte aber wiederholt gezeigt werden, dass Menschen dann besonders anfällig für die negativen Effekte von Stereotypaktivierung sind, wenn sie eine Domäne besonders wertschätzen ( $z$. B. Aronson et al., 1999; Cadinu et al., 2003; Keller, 2007; Levy, 1996). Daraus kann man ableiten, dass sowohl Schüler(innen) als auch Studierende, für welche der akademische Kontext von hoher Relevanz ist und die in diesem Bereich sehr gute Leistungen erbringen, vermehrt unter den Effekten negativer Stereotype leiden müssten (Worrell, 2009). Die 
wenigen empirischen Untersuchungen, die Stereotype Threat bei besonders begabten Schüler(innen) und Studierenden untersuchten, konnten diese Annahmen jedoch nicht bestätigen (Hargreaves, Homer \& Swinnerton, 2008; Walsh, Hickey \& Duffy, 1999). Aufgrund der dürftigen und widersprüchlichen empirischen Befunde kann man beim aktuellen Forschungsstand keine eindeutige Aussage über die Bedeutung von Stereotype Threat für Personen mit besonders günstigen Lernvoraussetzungen ableiten.

\section{Emotionsfokussierte Interventionen im Kontext von Stereotype Threat bei ethnischen Minderheiten}

Wie bereits in der Einleitung angesprochen liegt die Vermutung nahe, dass Menschen mit Migrationshintergrund in Deutschland häufig negative Emotionen erleben. Dies ergibt sich aus mehreren Beobachtungen:

1) Da negative Stereotype über bestimmte Migrantengruppen in Deutschland weit verbreitet sind, ist davon auszugehen, dass Menschen mit Migrationshintergrund häufig mit negative Stereotypen konfrontiert werden - sowohl in Leistungssituationen, als auch im alltäglichen Miteinander. Dies sollte für unterschiedliche Migrantengruppen unterschiedlich stark zutreffen, je nach dem in welchem Maß negative Stereotype über die jeweilige Gruppe verbreitet sind. So hat die Forschung zum Beispiel gezeigt, dass es vor allem über türkisch-stämmige Migranten sehr viele negative Stereotype gibt (z. B. Kahraman \& Knoblich, 2000). Andere Gruppen, wie Kinder und Jugendliche aus dem ehemaligen Jugoslawien sind hingegen erfolgreicher im deutschen Bildungssystem als türkisch-stämmige Migranten (z. B. Kristen, 2002), und haben somit wahrscheinlich auch weniger mit negativen Stereotypen zu kämpfen.

2) Familien mit Migrationshintergrund haben häufig einen niedrigen sozio-ökonomischen Status. Familien mit geringem Einkommen sind im alltäglichen Leben einer Vielzahl von stresserzeugenden Ereignissen (d.h. Stressoren) ausgesetzt (z. B. die Familie kann das Geld für eine Klassenfahrt des Kindes nicht aufbringen; BrooksGunn, Dundan, \& Aber, 1997). Diese Stressoren führen zu wiederholtem Erleben negativer Emotionen, die in irgendeiner Form verarbeitet bzw. bewältigt werden müssen.

In Bezug auf die zuvor dargestellte Forschung zu Stereotype Threat lässt sich festhalten, dass Emotionen als Mediatoren des Zusammenhangs zwischen der Aktivierung eines negativen Stereotyps und dessen Konsequenzen für Leistung und Motivation eine bedeutende Rolle spielen - insbesondere negative Emotionen standen bisher im Zentrum der Forschungsarbeiten (siehe Abbildung 13 und Abschnitt „Mediatoren“). Jenseits ihrer Funktion als Mediationsvariable ist anzunehmen, dass häufig erlebte negative Emotionen im Kontext von Stereotype Threat (z. B. Angst, Ärger, Hoffnungslosigkeit, Scham) 
zu generalisierten negativen affektiven Einstellungen bezüglich der stereotypisierten Domäne führen (kumulative Effekte). Beispielsweise kann bei ethnischen Minderheiten eine häufige Konfrontation mit dem Stereotyp "Türken sind weniger schlau als Deutsche" zu generalisierter Angst gegenüber bestimmten Leistungsdomänen führen. Diese generalisierte Angst hat wiederum Auswirkungen auf das Lernverhalten in dieser Domäne (z. B. Einsatz rigider Strategien), zukünftige Leistungen (z. B. Schulnote), Berufs- und Studienentscheidungen ( $z$. B. kein Studium beginnen) sowie lebenslanges Lernen ( $z$. B. "Abhaken" des Faches oder der akademischen Domäne im Allgemeinen nach der Schulzeit; Goetz \& Hall, in press; Pekrun, 2006; Pekrun, Goetz, Titz, \& Perry, 2002).

Doch müssen negative Emotionen, die aus dem Erleben von Stereotype Threat oder dem Erleben anderer Stressoren (z. B. die Familie kann das Geld für eine Klassenfahrt nicht aufbringen) resultieren, nicht immer einfach hingenommen werden - es gibt eine Reihe von Möglichkeiten ihrer Regulation. Strategien der Emotionsregulation wurden primär im Hinblick auf den Umgang mit Angst entwickelt; im Lern- und Leistungskontext vor allem bezüglich Prüfungsangst (Pekrun \& Götz, 2006; Zeidner, 1998, 2007). Doch auch jenseits der Angst gibt es mittlerweile eine relativ umfangreiche Literatur zur Emotionsregulation (z. B. Gross, 2007; Skinner, Edge, Altman, \& Sherwood, 2003). Eine zentrale und sehr häufig in der Literatur aufgegriffene Kategorisierung von Formen der Emotionsregulation basiert auf Forschungsarbeiten zum transaktionalen Stressmodell von R. S. Lazarus (vgl. Lazarus \& Folkman, 1984) und seinen Varianten (z. B. Spielberger \& Vagg, 1995). Hier werden problem-, emotions- und meidensorientierte Formen der Regulation unterschieden (vgl. Rost \& Schermer, 1987; Zeidner \& Endler, 1996). Diese Formen der Emotionsregulation und ihre mögliche Anwendung werden im Folgenden im Detail dargestellt.

\section{Problemorientierte Regulation}

Problemorientierte Regulation besteht aus aktiven Maßnahmen zur Änderung der Situation, in der negative Emotionen entstehen. Eine solche Regulation umfasst den gezielten Einsatz von metakognitiven, kognitiven und ressourcenorientierten Strategien, anhand derer Situationen, in welchen negative Emotionen erlebt werden, real oder in ihrer subjektiven Wahrnehmung verändert werden können. Was metakognitive Strategien anbelangt, so können beispielsweise Situationen, bei denen u. a. in Folge der Aktivierung negativer Stereotype negative Emotionen wie Angst und Hoffnungslosigkeit auftreten, reflektiert und die der Emotionsentstehung zugrunde liegenden Prozesse analysiert werden (z. B. Ausmaß an Gruppenidentifikation und ihre Wirkung auf Selbstkonzept und Emotionen). Wissen über Mechanismen und Prozesse im Kontext von Stereotype Threat ist diesbezüglich von zentraler Bedeutung und kann zu einer veränderten Sicht potenzieller Stereotype-Threat-Situationen führen (siche Johns, Schmader, \& Martens, 2005). Was kognitive Strategien anbelangt, so ist es beispielsweise möglich, sich 
auf Leistungssituationen durch den Einsatz effektiver Lern- und Arbeitsstrategien (siehe Boekaerts, Pintrich, \& Zeidner, 2005; Mandl \& Friedrich, 2006) intensiv vorzubereiten, um sich dadurch in der konkreten Situation und auch schon im Vorfeld selbstsicherer zu fühlen - und damit negativen Emotionen vorzubeugen (siehe Studien zu Self-affirmation [Selbstbestätigung] im Kontext von Stereotype Threat; z. B. Cohen, Garcia, Apfel, \& Master, 2006; Martens, Johns, Greenberg, \& Schimel, 2006). Zu mehr Selbstsicherheit dürften auch ressourcenbezogene Strategien führen, wie beispielsweise das Einrichten einer günstigen Lernumgebung, genügend Zeit und Anstrengung für die Vorbereitung auf die Leistungssituation zu verwenden und sich bei auftretenden oder antizipierten Problemen Unterstützung von anderen zu suchen oder sich entsprechende Hilfsmaterialien zu besorgen. Auch wenn problemorientierte Regulation generell als positiv zu bewerten ist, so sind auch potenziell negative Wirkungen ihres Einsatzes in Rechnung zu stellen (z. B. Verlust von Freunden bei sehr zeitintensiven Prüfungsvorbereitungen).

\section{Emotionsorientierte Regulation}

Emotionsorientierte Regulation zielt auf eine direkte Veränderung negativer Emotionen, d.h. sie fokussiert im Gegensatz zur problemorientierten Regulation weniger die Ursachen negativer Emotionen sondern diese selbst. Beispiele sind (a) der Einsatz von Entspannungstechniken (z. B. progressive Muskelrelaxation), (b) Alkohol-, Nikotin- und Medikamentenkonsum (z. B. Relaxantien), (c) das Zulassen und Akzeptieren eines gewissen Ausmaßes an negativen Emotionen, um dadurch z. B. sich-selbst-intensivierende Angstspiralen (z. B. Phobophobie, „Angst vor der Angst“) zu durchbrechen („sekundäre Kontrolle“; Rothbaum, Weisz \& Snyder, 1982) und (d) die Erzeugung affektiver Zustände, die mit negativen Emotionen inkompatibel sind (z. B. Freude durch Humor, Musik, Ironie). Bei einigen der Strategien sind selbstverständlich gesundheits- und leistungsschädliche Nebenwirkungen bei der Kosten-Nutzen-Rechnung im Hinblick auf ihren Einsatz zu berücksichtigen. Es erscheint plausibel anzunehmen, dass in Leistungssituationen, in denen negative Stereotype aktiviert werden, die direkte Regulation negativer Emotionen positive Effekte haben kann. Allerdings liegen bislang noch keine Studien vor, die diese These empirisch bestätigen.

\section{Meidensorientierte Regulation}

Während bei problemorientierter Regulation versucht wird, das Entstehen negativer Emotionen durch eine Modifikation der emotionsinduzierenden Situation zu verhindern und bei emotionsorientierter Regulation der Versuch unternommen wird, bereits vorhandene negative Emotionen zu reduzieren, zielt die meidensorientierte Regulation darauf ab, sich Situationen erst gar nicht auszusetzen, in denen negative Emotio- 
nen hervorgerufen werden könnten. Meidensorientierte Regulation besteht somit aus der Vermeidung einer Konfrontation mit einer (potenziell) bedrohlichen Situation bzw. einer behavioralen oder mentalen Flucht aus dieser, so dass negative Emotionen weniger oder erst gar nicht auftreten. Beispiele sind (a) Absentismus oder Abbruch der Tätigkeit, (b) Prokrastination (d.h. Aufschieben von Tätigkeiten) und überlange Pausen, sowie (c) mentale Ablenkung und Konzentration auf situationsirrelevante Aspekte (z.B. Tagträume, mentale Planung zukünftiger Aktivitäten). Verhaltensweisen dieser Art können zu einer situativen Reduktion negativer Emotionen führen. Allerdings kann es sich dabei auch um eine Verdrängung subjektiv erlebter negativer Emotionen handeln, die mit einer Erhöhung unbewusster, physiologisch nachweisbarer emotionaler Erregung „erkauft" wird (vgl. Spangler, Pekrun, Kramer \& Hofmann, 2002). Meidensorientierte Formen der Regulation wirken sich in der Regel negativ auf Wissenserwerb und Leistung aus. Dennoch sind sie keineswegs pauschal als dysfunktional zu bezeichnen. So kann sich beispielsweise der Abbruch einer stark überfordernden Tätigkeit oder eines permanent überfordernden Studiums durchaus im Hinblick auf die Genese des Selbstkonzepts und auch biografisch positiv auswirken. Im Kontext von Stereotpye Threat kann es beispielsweise durchaus angebracht sein, wenn Menschen mit Migrationshintergrund antizipierte "Threat-Situationen“ zumindest vorübergehend meiden, bis sie sich ihnen durch entsprechende Vorbereitungen (s. problemorientierte Regulation) gewachsen fühlen.

\section{Abgrenzung der drei Kategorien voneinander und Ausblick}

Eine Zuordnung von Regulationsverhalten zu den drei genannten Kategorien ist häufig nicht eindeutig möglich, sondern z. T. von der Perspektive abhängig (Pekrun \& Götz, 2006). So ist z. B. der Einsatz von Entspannungstechniken aus der Perspektive einer Reduktion von Stress (u. a. in Folge der Aktivierung negativer Stereotype) als emotionsorientierte Regulation anzusehen. Stellt man aber in Rechnung, dass entspannungsbedingte Stressreduktion der Leistungssteigerung in der entsprechenden Situation dienen kann, handelt es sich gleichzeitig auch um problemorientierte Regulation. Doch selbst wenn die drei Kategorien z. T. nicht trennscharf sind, so können sie doch sehr hilfreich im Hinblick auf die Verdeutlichung unterschiedlicher Möglichkeiten der Reduzierung negativer Emotionen sein. Zentral ist, dass man negativen Emotionen im Kontext von Stereotype Threat durchaus auf unterschiedliche Art und Weise begegnen kann. Da es relativ umfangreiche Literatur und empirische Evidenz zur Wirksamkeit der Emotionsregulation gibt, war es ein Ziel dieses Beitrags, einen elaborierten Ansatzpunkt zur Reduzierung der negativen Effekte der Aktivierung negativer Stereotype aufzuzeigen. Entsprechend der in Abbildung 13 dargestellten Mechanismen gibt es selbstverständlich noch weitere Interventionsmöglichkeiten, die u. a. bei Martiny \& Götz (2011) ausgeführt sind. Auf der Basis theoretischer Annahmen und empirischer Befunde Interventions- 
programme im Kontext von Stereotype Threat zu generieren, könnte einen bedeutsamen Beitrag zur Reduzierung ethnisch-kultureller Leistungsdisparitäten in Deutschland darstellen.

\section{Literatur}

Ambady, N., Shih, M., Kim, A., \& Pittinsky, T. (2001). Stereotype susceptibility in children: Effects of identity activation on quantitative performance. Psychological Science, 12(5), 385-390.

Aronson, J. (2002). Stereotype threat: Contending and coping with unnerving expectations. In J. Aronson (Ed.), Improving academic performance - Impact of psychological factors on education (pp. 281-299). Academic Press: London.

Aronson, J., Lustina, M., Good, C., Keough, K., Steele, C., \& Brown, J. (1999). When White men can't do math: Necessary and sufficient factors in stereotype threat. Journal of Experimental Social Psychology, 35(1), 29-46.

Beilock, S., Rydell, R., \& McConnell, A. (2007). Stereotype threat and working memory: Mechanisms, alleviation, and spillover. Journal of Experimental Psychology: General, 136(2), 256-276.

Berglas, S., \& Jones, E. (1978). Drug choice as a self-handicapping strategy in response to noncontingent success. Journal of Personality and Social Psychology, 36(4), 405-417.

Blascovich, J., Spencer, S., Quinn, D., \& Steele, C. (2001). African Americans and high blood pressure: The role of stereotype threat. Psychological Science, 12(3), 225-229.

Boekaerts, M., Pintrich, P., \& Zeidner, M. (Eds.), (2005). Handbook of Self-Regulation. San Diego: Academic Press.

Bosson, J., Haymovitz, E., \& Pinel, E. (2004). When saying and doing diverge: The effects of stereotype threat on self-reported versus non-verbal anxiety. Journal of Experimental Social Psychology, 40(2), 247-255.

Branscombe, N. R., Ellemers, N., Spears, R., \& Doosje, B. (1999) The context and content of social identity threat. In N. Ellemers, R. Spears, \& B. Doosje (Eds.), Social Identity: Context, commitment, Content. Oxford: Blackwell.

Branscombe, N. R., Schmitt, M. T., \& Harvey, R. D. (1999). Perceiving pervasive discrimination among African Americans: Implications for group identification and well-being. Journal of Personality and Social Psychology, 77, 135-149.

Brooks-Gunn, J., Duncan, G. L., \& Aber, J. L. (1997). Neighborhood poverty: Contexts and consequences for children. New York: Russell Sage Foundation.

Cadinu, M., Maass, A., Frigerio, S., Impagliazzo, L., \& Latinotti, S. (2003). Stereotype threat: The effect of expectancy on performance. European Journal of Social Psychology, 33(2), $267-285$.

Cadinu, M., Maass, A., Rosabianca, A., \& Kiesner, J. (2005). Why Do Women Underperform Under Stereotype Threat? Evidence for the Role of Negative Thinking. Psychological Science, 16(7), 572-578.

Carlson, R. A. (1997). Experienced cognition. Mahwah, NJ: Erlbaum.

Cohen, G., Garcia, J., Apfel, N., \& Master, A. (2006). Reducing the Racial Achievement Gap: A Social-Psychological Intervention. Science, 313(5791), 1307-1310. 
Croizet, J., \& Claire, T. (1998). Extending the concept of stereotype and threat to social class: The intellectual underperformance of students from low socioeconimic backgrounds. Personality and Social Psychology Bulletin, 24(6), 588-594.

Croizet, J., Després, G., Gauzins, M., Huguet, P., Leyens, J., \& Méot, A. (2004). Stereotype Threat Undermines Intellectual Performance by Triggering a Disruptive Mental Load. Personality and Social Psychology Bulletin, 30(6), 721-731.

Davies, P., Spencer, S., Quinn, D., \& Gerhardstein, R. (2002). Consuming images: How television commercials that elicit stereotype threat can restrain women academically and professionally. Personality and Social Psychology Bulletin, 28(12), 1615-1628.

Davis, C., Aronson, J., \& Salinas, M. (2006). Shades of Threat: Racial Identity as a Moderator of Stereotype Threat. Journal of Black Psychology, 32(4), 399-417.

Goetz, T. \& Hall, N. (in press). Emotion and achievement in the classroom. In J. Hattie and E. Anderman (Eds.), International Handbook on Student Achievement. London: Routledge Publishers.

Gross, J. J. (2007). Handbook of emotion regulation. New York: The Guilford Press.

Hargreaves, M., Homer, M., \& Swinnerton, B. (2008). A comparison of performance and attitudes in mathematics amongst the ,gifted.' Are boys better at mathematics or do they just think they are?. Assessment in Education: Principles, Policy \& Practice, 15(1), 19-38.

Huguet, P., \& Régner, I. (2007). Stereotype threat among schoolgirls in quasi-ordinary classroom circumstances. Journal of Educational Psychology, 99(3), 545-560.

Institut für Sozialforschung und Gesellschaftspolitik (2009). Integration in Deutschland - Erster Integrationsindikatorenbericht: Erprobung des Indikatorensets und Bericht zum bundesweiten Integrationsmomitoring. Retrieved from http://www.isg-institut.de

Jetten, J., Branscombe, N.R., Schmitt, M.T., \& Spears, R. (2001). Rebels with a cause: Group identification as a response to perceived discrimination from the mainstream. Personality and Social Psychology Bulletin, 27(9), 1204-1213.

Johns, M., Schmader, T., \& Martens, A. (2005). Knowing Is Half the Battle: Teaching Stereotype Threat as a Means of Improving Women's Math Performance. Psychological Science, 16(3), 175-179.

Kahraman, B. B., \& Knoblich, G. (2000). ,Stechen statt Sprechen': Valenz und Aktivierbarkeit von Stereotypen über Türken. Zeitschrift für Sozialpsychologie, 31(1), 31-43.

Keller, J. (2002). Blatant stereotype threat and women's math performance: Self-handicapping as a strategic means to cope with obtrusive negative performance expectations. Sex Roles, 47(3-4), 193-198.

Keller, J. (2007). Stereotype threat in classroom settings: The interactive effect of domain identification, task difficulty and stereotype threat on female students' maths performance. British Journal of Educational Psychology, 77(2), 323-338.

Keller, J., \& Dauenheimer, D. (2003). Stereotype threat in the classroom: Dejection mediates the disruptiing threat effect on women's math performance. Personality and Social Psychology Bulletin, 29(3), 371-381.

Koch, S., Müller, S., \& Sieverding, M. (2008). Women and computers. Effects of stereotype threat on attribution of failure. Computers \& Education, 51(4), 1795-1803.

Kray, L., Thompson, L., \& Galinsky, A. (2001). Battle of the sexes: Gender stereotype confirmation and reactance in negotiations. Journal of Personality and Social Psychology, 8o(6), 942-958.

Krendl, A., Richeson, J., Kelley, W., \& Heatherton, T. (2008). The negative consequences of threat: A functional magnetic resonance imaging investigation of the neural mechanisms underlying women's underperformance in math. Psychological Science, 19(2), 168-175. 
Kristen, C. (2002). Hauptschule, Realschule oder Gymnasium? Ethnische Unterschiede am ersten Bildungsübergang. Kölner Zeitschrift für Soziologie und Sozialpsychologie, 54(3), 534-552.

Lazarus, R. S. \& Folkman, S. (1984). Stress, appraisal, and coping. New York: Springer.

Levy, B. (1996). Improving memory in old age through implicit self-stereotyping. Journal of Personality and Social Psychology, 71(6), 1092-1107.

Leyens, J., Désert, M., Croizet, J., \& Darcis, C. (2000). Stereotype threat: Are lower status and history of stigmatization preconditions of stereotype threat?. Personality and Social Psychology Bulletin, 26(10), 1189-1199.

Mandl, H. \& Friedrich, H. F. (2006) (Hrsg.). Handbuch Lernstrategien. Göttingen: Hogrefe.

Martens, A., Johns, M., Greenberg, J., \& Schimel, J. (2006). Combating stereotype threat: The effect of self-affirmation on women's intellectual performance. Journal of Experimental Social Psychology, 42(2), 236-243.

Martiny, S. E., \& Götz, T. (2011). Stereotype Threat in Lern- und Leistungssituationen: Wirkungen auf Leistung und Motivation. In M. Dresel, \& L. Laemmle (Eds.). Motivation, Selbstregulation und Leistungsexzellenz.

Martiny, S. E., Roth, J. \& Deaux, K. (2011). The role of domain identification and arousal in stereotype threat. Manuscript in preparation.

Martiny, S. E., Roth, J., Jelenec, P., Steffens, M., \& Croizet, J. (2012). When a new group identity does harm on the spot: Stereotype threat in newly created groups. European Journal of Social Psychology.

McGlone, M., \& Aronson, J. (2006). Stereotype threat, identity salience, and spatial reasoning. Journal of Applied Developmental Psychology, 27(5), 486-493.

Nussbaum, A., \& Steele, C. (2007). Situational disengagement and persistence in the face of adversity. Journal of Experimental Social Psychology, 43(1), 127-134.

Osborne, J. (1997). Race and academic disidentification. Journal of Educational Psychology, $89(4), 728-735$.

Osborne, J. (2007). Linking Stereotype Threat and Anxiety. Educational Psychology, 27(1), $135-154$.

Osborne, J., \& Walker, C. (2006). Stereotype Threat, Identification with Academics, and Withdrawal from School: Why the most successful students of colour might be most likely to withdraw. Educational Psychology, 26(4), 563-577.

Pekrun, R. \& Götz, T. (2006). Emotionsregulation: Vom Umgang mit Prüfungsangst. In H. Mandl \& H. F. Friedrich (Hrsg.), Handbuch Lernstrategien (S. 248-258). Göttingen: Hogrefe.

Pekrun, R. (2006). The control-value theory of achievement emotions: Assumptions, corollaries, and implications for educational research and practice. Educational Psychology Review, 18, 315-341.

Pekrun, R., Goetz, T., Titz, W., \& Perry, R. P. (2002). Academic emotions in students' self-regulated learning and achievement: A program of qualitative and quantitative research. Educational Psychologist, 37(2), 91-105.

Prenzel, M. u. a. (Hrsg.). (2004). PISA 2003. Der Bildungsstand der Jugendlichen in DeutschlandErgebnisse des zweiten internationalen Vergleichs. Waxmann: Münster.

Prenzel, M. u. a. (Hrsg.). (2007). PISA 2006. Die Ergebnisse der dritten internationalen Vergleichsstudie. Waxmann: Münster.

Rahhal, T., Hasher, L., \& Colcombe, S. (2001). Instructional manipulations and age differences in memory: Now you see them, now you don't. Psychology and Aging, 16(4), 697-706. 
Rost, D.H. \& Schermer, F. J. (1987). Emotion and cognition in coping with test anxiety. Communication and Cognition, 20, 225-244.

Rothbaum, F., Weisz, J.R. \& Snyder, S.S. (1982). Changing the world and changing the self: A two-process model of perceived control. Journal of Personality and Social Psycholo$g y, 42,5-37$.

Rydell, R., McConnell, A., \& Beilock, S. (2009). Multiple social identities and stereotype threat: Imbalance, accessibility, and working memory. Journal of Personality and Social Psycho$\log y, 96(5), 949-966$.

Schmader, T. (2002). Gender identification moderates stereotype threat effects on women's math performance. Journal of Experimental Social Psychology, 38(2), 194-201.

Schmader, T., \& Johns, M. (2003). Converging Evidence That Stereotype Threat Reduces Working Memory Capacity. Journal of Personality and Social Psychology, 85(3), 440-452.

Schmader, T., Johns, M., \& Forbes, C. (2008). An integrated process model of stereotype threat effects on performance. Psychological Review, 115(2), 336-356.

Shih, M., Pittinsky, T., \& Ambady, N. (1999). Stereotype susceptibility: Identity salience and shifts in quantitative performance. Psychological Science, 10(1), 80-83.

Shih, M., Pittinsky, T., \& Trahan, A. (2006). Domain-specific Effects of Stereotypes on Performance. Self and Identity, 5(1), 1-14.

Skinner, E. A., Edge, K., Altman, J., \& Sherwood, H. (2003). Searching for the structure of coping: A review and critique of category systems for classifying ways of coping. Psychological Bulletin, 129, 216-269.

Spangler, G., Pekrun, R., Kramer, K. \& Hofmann, H. (2002). Students' emotions, physiological reactions, and coping in academic exams. Anxiety, Stress and Coping, 15, 413-432.

Spencer, B., \& Castano, E. (2007). Social class is dead. Long live social class! Stereotype threat among low socioeconomic status individuals. Social Justice Research, 20(4), 418-432.

Spencer, S., Steele, C., \& Quinn, D. (1999). Stereotype threat and women's math performance. Journal of Experimental Social Psychology, 35(1), 4-28.

Spielberger, C. D. \& Vagg, P. R. (1995). Test anxiety: A transactional process. In C. D. Spielberger \& P. R. Vagg (Eds.), Test anxiety: Theory, assessment, and treatment (pp. 3-14).

Stangor, C., Carr, C., \& Kiang, L. (1998). Activating stereotypes undermines task performance expectations. Journal of Personality and Social Psychology, 75(5), 1191-1197.

Steele, C. (1992). Race and the schooling of Black Americans. The Atlantic Monthly, 68-78.

Steele, C. (1997). A threat in the air: How stereotypes shape intellectual identity and performance. American Psychologist, 52(6), 613-629.

Steele, C., \& Aronson, J. (1995). Stereotype threat and the intellectual test performance of African Americans. Journal of Personality and Social Psychology, 69(5), 797-811.

Stone, J. (2002). Battling doubt by avoiding practice: The effects of stereotype threat on self-handicapping in white athletes. Personality and Social Psychology Bulletin, 28(12), 1667-1678.

Stone, J., Lynch, C., Sjomeling, M., \& Darley, J. (1999). Stereotype threat effects on Black and White athletic performance. Journal of Personality and Social Psychology, 77(6), 1213-1227.

Tajfel, H. \& Turner, J. (1979). An integrative theory of intergroup conflict. In M. A. Hogg \& D. Abrams (Eds.), Intergroup relations: Essential readings (pp. 94-109). Psychology Press.

von Hippel, W., von Hippel, C., Conway, L., Preacher, K., Schooler, J., \& Radvansky, G. (2005). Coping With Stereotype Threat: Denial as an Impression Management Strategy. Journal of Personality and Social Psychology, 89(1), 22-35.

Walsh, M., Hickey, C., \& Duffy, J. (1999). Influence of item content and stereotype situation on gender differences in mathematical problem solving. Sex Roles, 41(3-4), 219-240. 
Worrell, F. (2009). What does gifted mean? Personal and social identity perspectives on giftedness in adolescence. The development of giftedness and talent across the life span (pp. 131-152). Washington, DC US: American Psychological Association.

Wout, D., Danso, H., Jackson, J., \& Spencer, S. (2008). The many faces of stereotype threat: Group- and self-threat. Journal of Experimental Social Psychology, 44(3), 792-799.

Yopyk, D., \& Prentice, D. (2005). Am I an athlete or a student? Identity salience and stereotype threat in student-athletes. Basic and Applied Social Psychology, 27(4), 329-336.

Zeidner, M. \& Endler, N. (Eds.). (1996). Handbook of coping. New York: Wiley.

Zeidner, M. (1998). Test anxiety: The state of the art. New York, NY US: Plenum Press.

Zeidner, M. (2007). Test anxiety: Conceptions, findings, conclusions. In P. A. Schutz \& R. Pekrun (Eds.), Emotion in education (pp. 165-184). San Diego: Academic Press. 